\title{
Process monitoring for continuous improvement
}

\author{
V.J. Thomson \\ Werner Graupe Chair in Manufacturing Automation \\ Department of Mechanical Engineering \\ McGill University \\ 817 Sherbrooke Street West, Montreal, Quebec, Canada, H3A 2 K6 \\ tel: 1-514-398-2597, fax: 1-514-398-7365, me60@musica.mcgill.ca
}

\begin{abstract}
The combined trends of increasing levels of knowledge work, but declining productivity of knowledge workers have hampered efforts to improve overall corporate productivity in the industrialized world. The active management of knowledge work processes promises to provide a significant improvement in organization wide productivity. Process monitoring, a tool which measures realtime process performance, permits proactive management of processes and enables their continuous improvement. The design of a process monitoring tool is described along with industrial projects which demonstrate its usage.
\end{abstract}

Keywords

Process monitoring, business process reengineering, productivity

\section{INTRODUCTION}

For more than a decade, the productivity of knowledge workers has been going down rather than up. U.S.A. labour statistics from 1980 to 1990 reveal that blue collar productivity rose $28 \%$, while at the same time white collar productivity declined $3 \%$ (see Table 1 ). In addition, the relative contribution of knowledge work to product value is increasing continuously, while material content and the cost of production have decreased. According to Miller and Vollmann (1985), "The indirect work embodied in logistics, balancing, quality and change transactions now accounts for the lion's share of value added in most production based industries." In the electronics industry, overhead is as high as $80 \%$ (Miller, 1985). Increased overhead or knowledge worker levels are also a fact in service organizations in addition to product oriented industries. Overhead distribution in the electronics industry is given in Table 2; the majority of these costs are salaries and support for knowledge work. 
Table 1 Worker productivity comparison (Thurow, 1992)

Real output grew $30 \%$ in the U.S.A. (1980-1990).

\begin{tabular}{lcc}
\hline Growth & Blue collar $(\%)$ & White collar $(\%)$ \\
\hline Employment & 2 & 33 \\
Productivity & 28 & -3 \\
\hline
\end{tabular}

Table 2 Manufacturing overhead costs in the electronics industry (Miller, 1985)

\begin{tabular}{lll}
\hline Materials overhead & $33 \%$ & (procurement, off floor handling, coordination) \\
General and administrative & $20 \%$ & (personnel, accounting, management) \\
Facilities and equipment & $20 \%$ & (capital, insurance, energy (4\% of total cost)) \\
Engineering & $15 \%$ & (design, planning) \\
Indirect labour & $12 \%$ & (maintenance, quality control) \\
\hline
\end{tabular}

The problem of white collar (knowledge) worker productivity is present in all economies of the world. Shintaro Hori (1993) in an article on Japan's white collar economy states that: "Companies must reorder their functional priorities, shifting their focus from production and product development to white collar work." The article points out that the disproportionate growth of overhead in the sales, general and administration category in Japanese companies from $29.2 \%$ (1984) to $33.5 \%$ (1992) is symptomatic of productivity problems. Hori proposes that industry must focus on process oriented functions to improve white collar productivity. These combined trends of increased levels of knowledge work, but declining productivity of knowledge workers have hampered efforts to improve overall corporate productivity in the industrialized world.

As well, the future will see more and more emphasis on process. With ready access to all forms of knowledge, what is starting to differentiate companies, and what will continue to differentiate them, is process knowledge. This is reinforced by Lester Thurow's (1993) statement in Head to Head that, "in the future sustainable competitive advantage will depend more on new process technologies and less on new product technologies." Process is a competitive advantage; it needs to be managed as a corporate asset.

Manufacturing has been highly successful in increasing productivity through commonly designed and executed processes. The same approach needs to be used for 'soft' or knowledge work processes, such as, design, engineering, procurement, distribution, etc. There is a need for a new culture based on process management which will enable the design, planning, and monitoring of all processes in an enterprise. This will lead to a significant improvement in organization wide productivity.

\section{PROCESS MONITORING}

Production systems in discrete and continuous manufacturing have operated for many years with highly defined processes and monitoring systems to deliver feedback for continuous improvement. Underlying principles and methods have been developed for these systems to provide the necessary techniques to allow appropriate process definition and process monitoring. The development of these principles and methods has allowed manufacturing industries to have continuous productivity improvement in their production systems. More 
importantly, continuous process improvement has also led to ever increasing levels of process complexity, which has meant better products and services, even while reducing overall cost structures.

Process monitoring needs to be developed for knowledge work processes. This will allow processes to be planned, measured and continuously improved. In addition, process monitoring promises to allow knowledge work to progress to higher levels of sophistication, and thus, produce better products and services, by assisting the active management of processes, as has been done on the shop floor. In fact, for 'soft' processes, process monitoring systems are even more imperative. For, in knowledge work much more initiative is allowed, and indeed, expected on the part of workers. This means that knowledge work processes are less rigidly defined, and are expected to be highly adaptive to circumstances. Thus, the monitoring of processes must provide significant capabilities in terms of comparison to planned activities, continuous measurement of performance, and prediction of trends in order to manage processes better.

The use of statistical process control techniques, which are aimed at measuring variations in the characteristics of outputs or products of processes, can be used in some cases for monitoring of knowledge work processes. However, specific, statistically measurable outputs are not available for many processes, or in a lot of cases, the output which is easily measurable does not reflect very well the goals of the process, and is, therefore, not very useful in judging the quality of the process. Also, good metrics have not been developed for measuring the quality of the process itself. For example, good engineering process is measured by the output of an engineering group. If this has relatively high volume of somewhat comparable products, such as electronic circuit designs, process performance is implied from 'good' output; but, if it has low volume of not very comparable products, measurement of the performance of process is nearly impossible. A new basis for measurement is needed, and must focus on the effectiveness of the process itself, not on its output. The concept of process monitoring proposed here will provide this capability.

Process monitoring starts with the basic notion of comparing a real process to a model of the desired process in order to provide performance comparisons and feedback. The key idea is the measurement of process parameters, not necessarily to determine any characteristics of the product being made, but to measure the process itself. These parameters need to be easily measured to reflect how the process is doing, and to allow feedback for process control. Here, the principle of 'You can't manage what you don't measure' applies. Thus, process monitoring captures realtime measurements of processes, performs a comparison of these measurements with models of the processes, and then, provides feedback to workers for performance analysis and process redesign. The design of a process monitoring system presently being built is shown in Figure 1.

The major components of the process monitoring system are: a process modeller which facilitates the design and planning of processes; realtime process measurement (not shown) which gathers selected data from actual processes; a process comparison engine which compares the performance of real world processes to process models in realtime; a feedback generator which delivers reports on process performance; a tool kit which contains algorithms and procedures for measuring performance, analyzing process maps, and enabling some changes to processes for low level automation of process monitoring; and a simulator which can 'replay' real processes for process review as part of feedback. 


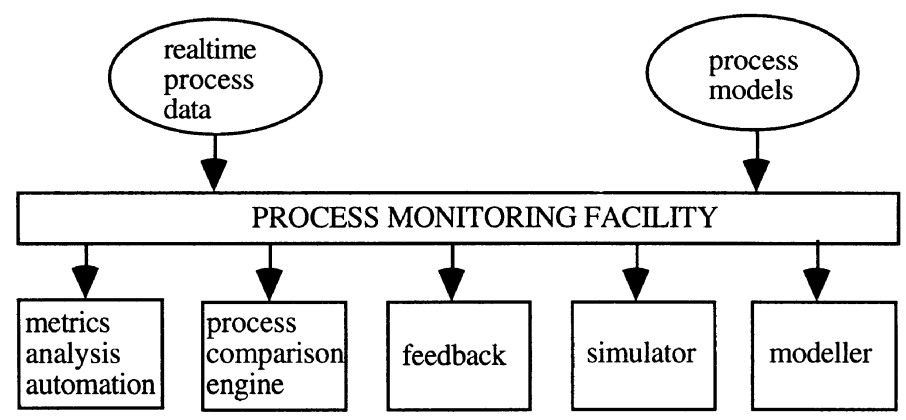

Figure 1 Process monitoring system.

For such a system, data acquisition techniques as well as suitable process modelling and process model simulation methods exist so that in creating process monitoring tools, the main item to be developed is a process comparison engine, i.e., the comparison of data and measurements from real processes with corresponding items in process models. In order to do process model to real process data comparisons, some of the major issues are: the determination of cause and effect in a many step process; the handling of comparison when data collected from real processes and data from process models are at different levels of granularity, i.e., where the levels of detail between the real process and its model are different; and the automatic testing of process rules to determine if they are followed and what are allowable exceptions. For process monitoring systems to work effectively, the systems must be able to traverse quickly large process maps with a large number of interconnections, and to link causes with effect in order to qualify and quantify feedback for process workers about process performance, assignment of process error, and the following or violation of process rules. A particular issue is that due to cost, data cannot be collected for all steps in every process; therefore, techniques are being developed for determining cause and effect relationships in processes for the case of sparse measurements.

As mentioned earlier, process modellers do exist. For process modelling and process simulation, FirstSTEP, a commercial product by Interfacing Technologies Inc., is being used. It is built in object technology (ParcPlace: VisualWorks, based on Smalltalk). For use in process monitoring, FirstSTEP has the advantage that it considers tasks from the process point of view rather than the functional point of view (Pardasani, 1992). This allows simple definition of processes and a simple basis for comparison of processes. FirstSTEP can also simulate models of processes.

However, the design of a simulator for process monitoring needs to be different than a simulator for process models. It is impractical to measure all the data for real processes; therefore, a simulator needs to be able to 'replay' real data while simultaneously considering models of the processes in order to interpolate between measured points and fill in missing information. Intelligent agent technology has been developed which can operate in realtime. Methods are being designed to be added to these agents in order to do the necessary interpretation of models and interpolation of data. 
For easy analysis of monitored processes, it is imperative that a good tool kit be developed which contains process metrics and analysis tools. Several types of metrics are needed which can indicate process performance, flexibility, cost, complexity, coordination, etc. Many concepts exist such as, activity based costing, value added, on time delivery, and throughput; algorithms for these are being developed for incorporation into a process monitoring system. Other metrics, such as, an entropic measure for flexibility which has been considered for flexible manufacturing systems (Kumar, 1986) are being developed for use with all processes.

One of the important considerations for managing processes are interfaces, i.e., points at which items are handed off between departments and/or organizations. Process analysis tools are important, particularly, ones such as process connectivity, interface identification, as well as data volumes and timing at interfaces; all of which can assist in interface management.

Presenting feedback information is problematic. The mechanism needs to be simple for easy interpretation, yet sophisticated enough to allow viewing of large systems of processes. It needs to show which processes are not operating effectively or are in trouble, as well as indicating the upstream and downstream processes which can affect or be impacted by problem processes.

Since process monitoring is information intensive, methods for automating data gathering, analysis and reporting of feedback need to be part of a process tool kit. The methods are not for automating the process, but for simplifying process monitoring.

\section{METHODOLOGY}

At present, process monitoring concepts are being developed in a three step process:

1) design the major system components and the process tool kit using object technology;

2) develop and test the concepts in the laboratory using a process modeller/simulator; and

3 ) examine simple prototypes inside companies to test concepts further.

Simulations of process scenarios using data acquired from actual processes will also allow the determination of performance characteristics for processes, and thus, desirable features for process analysis tools and metrics. As stated above, the process modeller/simulator used is FirstSTEP. Process monitoring prototypes will be built using VisualWorks and Smalltalk. Object oriented technology has proven to be very effective for building applications which can handle great complexity, and at the same time provide interfaces which are very simple to use.

Intelligent agent technology is being studied to judge its usefulness in tracking the execution of processes and assignment of cause and effect relationships on the basis of realtime data collection, process metrics, and the parent-child relationships of process steps and lower level activities.

Realtime operation of a process monitoring system is deemed to be essential; the appropriate level of realtime response and analysis versus likely usage scenarios are being studied. The challenge of overall system design is to make it simple to use. The total system of process planning in addition to measuring and updating of process data must be transparent to those who operate processes; otherwise, process monitoring will not be adopted. 


\section{TEST CASE EXAMPLES}

There are many activities inside corporations which have a significant impact upon overall performance, and which are difficult to manage with existing systems. Examples are given below which demonstrate how process management and, in particular, process monitoring can provide a good means to better both performance and control in corporations.

\subsection{Managing corporate processes in a project environment}

The operation of many corporations can be characterized as the performance of projects, e.g., design/engineering companies, product/service development organizations, etc. Projects have different life times and are generally not related to each another, i.e., different deliverables, different customers or both. However, all of the projects make use of a small set of common processes inside the corporation. These processes are used at different times, and possibly, in different ways. It is presently being realized by many corporations that competitive advantage comes from support processes, not the ability to manage projects. In most cases, these processes contain the corporation's core competencies.

To manage projects corporations use management information systems to track time and cost by activity centre for projects. Process information is rarely captured; but, it is the performance of processes common to all projects which greatly affects project performance. These critical processes need to be actively managed. Process monitoring is the solution, i.e., measurement of key parameters which indicate good process/project performance, and systematic review so that both processes and projects are carried out effectively. In addition, feedback must be given to all process performers so that they can continuously improve the processes for the better execution of projects.

The benefits of process monitoring in this scenario are twofold. First, project mangers are able to influence the processes which have a direct bearing on project execution, and thus, improve project effectiveness. Secondly, process owners are able to improve processes and core competencies for overall corporate productivity improvement.

\subsection{Engineering specification approval}

Many companies bid on contracts singly or with a group of partners. For custom designed and engineered products, once the contract is awarded, there is a requirement to exchange engineering specifications about the to-be-delivered product with the customer, prime contractor and/or contract partners. This is quite common for the delivery of final products or subsystems for large items, such as bridges, ships, aircraft, hydro electric projects, buildings, etc.

The process for the exchange of engineering specifications has many problems: highly variable length of time, highly variable cost, changing circumstances for different projects, and changing specifications which can change production costs. The inability to estimate the length of time and cost to arrive at agreement on engineering specifications greatly impacts the ability to finalize design and to schedule production. Constant changes lead to rework for engineering calculations and product design. Also, one of the most significant difficulties is the changing nature of the problem from project to project; this leads to paralysis in trying to find a solution, since it appears that there needs to be a different solution for each project. 
Process management can assist in reducing the uncertainty associated with the process for engineering specification approval by designing a suitable process (Thomson, 1995). However, the major difficulty is not so much poor design of process, but the large number of interfaces both inside and outside the corporation across which flow exchanges of information. It is these interfaces for information exchange which determine the efficacy of the process. A process monitoring system is needed to track key parameters in order to measure process performance, especially in regards to the management of these interfaces, and to provide feedback to both sides of an interface to allow for knowledge about the state of the process and for continuous improvement of process.

An important concept in this case is that the design of the process framework for improved engineering specification approval must contain process monitoring, or at least provide for its later inclusion. For, process redesign alone with not guarantee good process performance; only the active management of interfaces through process monitoring will do this.

\section{CONCLUSION}

Knowledge work is making up a greater and greater share of work inside corporations in the industrialized world. To improve knowledge worker productivity, knowledge processes need to be actively managed. Process monitoring, i.e., the capture of realtime measurements of processes, the comparison of these measurements with data from process models, and the provision of feedback, promises to provide a tool which will permit proactive management of processes and also enable their continuous improvement.

There are many types of activities in corporations which can best be managed by the gathering and analysis of data about process, not product or service. Two such scenarios were discussed showing how a process monitoring tool can assist in this management. An important characteristic of such activities is that the processes contain key competencies which the corporation needs to manage well in order to operate effectively.

\section{REFERENCES}

Miller, J.G. and Vollmann, T.E. (1985) The hidden factory. Harvard Business Review, SepOct. 142-50.

Hori, S. (1993) Fixing Japan's white collar economy: a personal view. Harvard Business Review, Nov-Dec, 157-72.

Kumar, V. (1986) On measurement of flexibility in flexible manufacturing systems: an information-theoretic approach. Proceedings of the Second International Conference on Flexible Manufacturing Systems: Operations Research Models and Applications (ed. K.E. Stecke and R. Suri), 131-43.

Pardasani, A. and Chan, A. (1992) Enterprise model: a decision support tool for computer integrated manufacturing, Proceedings of the International Conference on Object Oriented Manufacturing Systems, Calgary, May, 182-7.

Thomson, V. (1995) Process management in manufacturing. Control Engineering Practice, (to be published Feb 1995).

Thurow, L. (1992) Head to Head. W. Morrow \& Co., New York. 


\section{BIOGRAPHY}

The author has spent 15 years at the National Research Council (Ottawa, Canada) working with industry on integrated manufacturing problems. He has recently been appointed to the Werner Graupe Chair in Manufacturing Automation at McGill University (Montreal) where he is doing research on process management. 